\title{
Ball release experiments on a centrifuge: misalignment between the buoyancy force and the axis of rotation
}

\author{
By U. RIEMENSCHNEIDER ${ }^{1}$ AND V. A. SHEREMET \\ ${ }^{1}$ Department of Physical Oceanography, Woods Hole Oceanographic Institution, \\ Woods Hole, MA 02543, USA \\ ${ }^{2}$ Graduate School of Oceanography, University of Rhode Island, Narragansett, RI 02882, USA.
}

(Received 18 February 2005 and in revised form 27 March 2006)

Motivated by work on tilted convection (Sheremet, J. Fluid Mech., vol. 506, 2004, p. 217), a set of experiments is presented here using the same set-up of a tilted tank attached to a rotating centrifuge with a $2.5 \mathrm{~m}$ arm. Within the tank small, almost neutrally buoyant, spheres are released, and their trajectories are recorded. Thus the forces acting on a sphere can be analysed in the case of misalignment between the buoyancy force and the axis of rotation.

The angles of descent characterizing the trajectory are compared with inviscid linear theory developed by Stewartson (Q. J. Math. Appl. Mech., vol. 6, 1953, p. 141), and the agreement is found to be good. The angles should be independent of the density anomaly of the spheres compared to their environment. Using the descent velocity from non-rotating experiments, the density of the spheres is estimated and used to determine the drag acting on them in the rotating experiments. It is found that the drag is up to $50 \%$ larger than expected from Stewartson's theory. The agreement is best, not for infinitesimal, but for small Rossby numbers. The results are consistent with observations recorded by Maxworthy (J. Fluid Mech., vol. 40, 1970, p. 453).

\section{Introduction}

The ball release experiments outlined in this work were motivated by laboratory experiments with tilted convective plumes on a centrifuge carried out by Sheremet (2004). These plume experiments were conducted to study the effect of the misalignment of gravity and the rotation axis on small convective cells. Such cells are argued to play an important role in the process of deep convection occurring in polar regions, like the Labrador, Greenland and Weddell Seas. In these regions the stratification of the water column is often observed to be very weak, meaning that the underlying deep water is only marginally denser than the overlying surface waters. In the winter months, when wind surface cooling is particularly effective, the surface waters may actually become denser and large patches of water of up to hundreds of kilometres across may be vertically mixed to a depth of 1 to 2 kilometres in periods of only a few weeks. Field observations in the Labrador Sea by Pickart, Torres \& Clarke (2002) have suggested that most of the mixing happens in smaller convective cells, with horizontal scales of less than 1 kilometre. It is the effect of both components of rotation on these cells that was studied by Sheremet (2004). The argument is that some of the convective motion may be aligned along the axis of rotation, rather than along the axis parallel to the action of the gravitational acceleration. This is due to 
the Taylor-Proudman theorem, which states that in slowly moving steady flow, the fluid velocity will be uniform along any line parallel to the axis of rotation.

In the experiments by Sheremet (2004), the plumes were produced by injecting slightly dense coloured salty water into a tank of freshwater mounted on a centrifuge, which was rotating at a constant angular velocity, and the formation of tilted Taylor columns ('Taylor ink-walls') was observed. Sheremet also found that for low Reynolds numbers the incoming jet of dyed fluid disintegrates into coherent blobs, which descend neither in the direction of the buoyancy force $\boldsymbol{g}_{\mathrm{e}}$ nor in the direction of rotation $\Omega$. Instead, they assume a trajectory at an angle which compares quite well with linear theory developed by Loper (2001).

It is this blob structure of the plume that sparked the idea of studying the behaviour of small spheres in the same experimental setup. We set out to test experimentally that both the linear viscous theory of Loper (2001), for the spherical fluid parcels, and the linear inviscid theory of Stewartson (1953), for solid spheres, give identical results for the trajectories. In the past, there have been a number of publications on experimental determination of forces acting on a sphere moving through a rotating fluid, e.g. Taylor (1921), Maxworthy (1970), Moll (1973), and Mason (1975). The experiments reported in this paper are special in the following three aspects: (i) The distance of the tank from the axis of rotation of the centrifuge is small compared to its size; therefore, we can assume that the forces acting on a sphere are uniform in space to a first approximation; (ii) A finite misalignment between the axis of rotation and the buoyancy force is produced by utilizing the centrifugal acceleration; (iii) We use almost neutrally buoyant spheres; this allows the drag, lift, and Coriolis forces, which depend linearly on velocity, to be combined in a single matrix form.

The experimental setup will be described in $\S 2$, then the physics of flow around spheres will be briefly reviewed in $\S 3$. In $\S 4$ the force balance on the particle will be outlined, and in $\S 5$ we briefly review the theory developed by Stewartson (1953). Following in $\S 6$ will be the presentation of the experimental results for the tilted experiments, as well as centre-rotating and non-rotating experiments, which were carried out for comparison. The difficulties encountered in determining the density of the nylon spheres used in the experiment are discussed separately in the Appendix. We will use the density estimates to deduce the drag on the spheres in a rotating environment in $\S 7$, and conclusions will be drawn in $\S 8$.

\section{Experimental setup}

A sketch of the apparatus is shown in figure 1. The experiments were carried out on a centrifuge consisting of a wooden frame extending $2.5 \mathrm{~m}$ in both directions away from the centre of the rotating table. A box-shaped Plexiglas tank of inner dimensions $29 \times 29 \times 50 \mathrm{~cm}$ (width $\times$ depth $\times$ height) was mounted either at one end of the centrifuge at a distance of $R=2.46 \mathrm{~m}$ from the centre of the table (for the tilted tank experiments, series $\mathrm{N}$ ) or in the centre (for the centre-rotating experiments, series $\mathrm{R}$, and non-rotating, series $\mathrm{S}$ ).

To conduct the tilted tank experiments (series $N$ ) weights were placed at the opposite end to keep the centrifuge in balance. The tank was attached with two pivots so that it could be tilted inward through an angle of $\alpha=30^{\circ}$ and the rotation rate was adjusted to $\Omega=1.517 \mathrm{~s}^{-1}$ according to

$$
\tan \alpha=\frac{\Omega^{2} R}{g}
$$




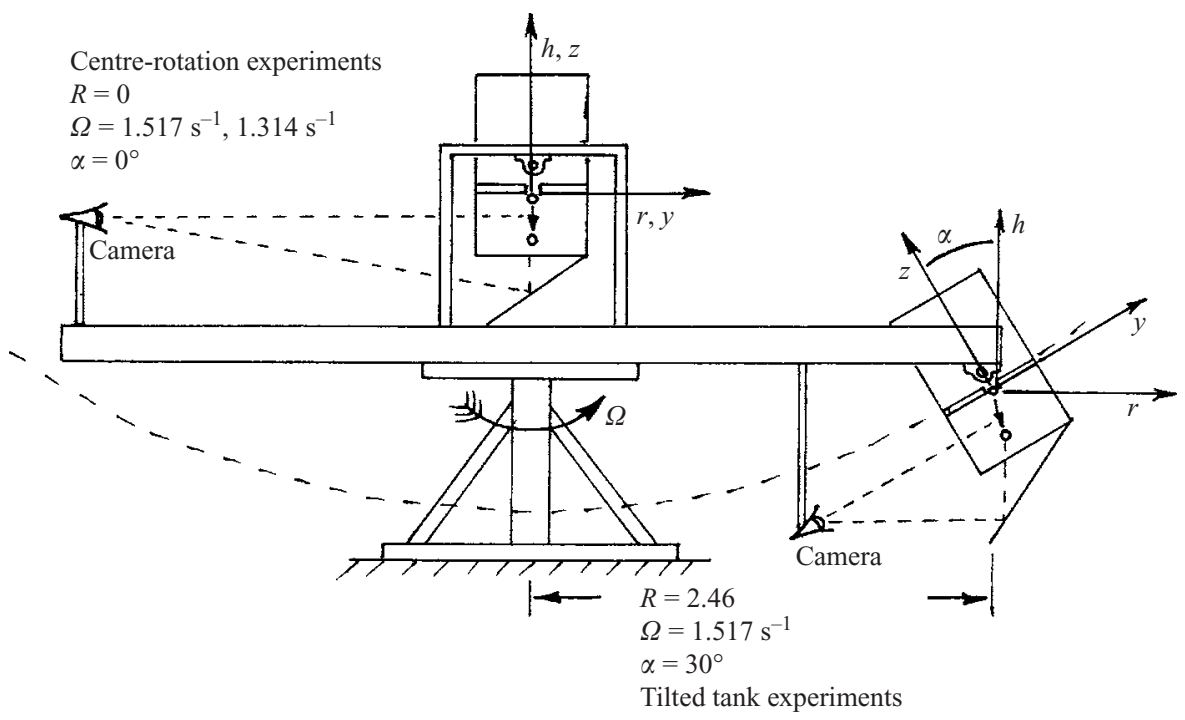

FIGURE 1. Sketch of the experimental setup.

(see Sheremet 2004, equation (2.4)), where $g=9.803 \mathrm{~m} \mathrm{~s}^{-2}$ is the gravitational acceleration. When the apparatus was spinning with the full angular velocity, the free surface of the water (as indicated by an equipotential parabolic surface shown by a dashed line in figure 1) was approximately parallel to the bottom of the tank. The tank was filled with enough water to ensure that the surface of the water was about $20 \mathrm{~cm}$ above the bottom when the tank was rotating. A lid was inserted into the tank such that the bottom $20 \mathrm{~cm}$ formed a body of fluid that was bounded by solid walls on all sides. On top of the lid a remotely controlled release mechanism for the balls was attached, which allowed them to be dropped through a hole at the centre of the lid. The mechanism consisted of a metal tube containing up to eight nylon balls of $1.270 \mathrm{~cm}(1 / 2 \mathrm{in}$.) diameter, a Plexiglas slide transferring the balls from the tube to the hole in the lid, and a solenoid pulling the slide. The diameter of the hole in the lid was only slightly larger than that of the ball. Therefore, due to increased friction as the ball travelled through the hole, the initial disturbance given to it by the release mechanism was dissipated, and the ball appeared at the exit from the hole with practically negligible velocity. The balls were marked with coloured lines around three geodesics perpendicular to each other in order to observe their possible spinning motion.

The balls (purchased from Small Parts, Inc.) were made of Nylon 6/6, which has approximate density of $\rho_{B}=1.115 \mathrm{~g} \mathrm{~cm}^{-3}$. As a working fluid we used aqueous sodium chloride (common salt) solution of slightly lower density, $\rho_{20}=$ $1.11456 \mathrm{~g} \mathrm{~cm}^{-3}$ (which was determined by densitometer with $10^{-5}$ precision at $20^{\circ} \mathrm{C}$ ) to allow the balls to sink slowly during the experiments. The typical density difference was $O\left(10^{-3} \mathrm{~g} \mathrm{~cm}^{-3}\right)$. Due to manufacturing imperfections, the density of each ball was slightly different resulting in different settling velocity. Some prepared balls were floating and were discarded. For presentation purposes we selected four and identified them by letters B5, B1, B2, B6 in order of increasing density as in Riemenschneider (2002). The temperature of the fluid during the experiments $T_{F}$ was within a $24^{\circ} \mathrm{C}$ $26^{\circ} \mathrm{C}$ range and within $0.5^{\circ} \mathrm{C}$ of the room air temperature. $T_{F}$ was recorded in order 


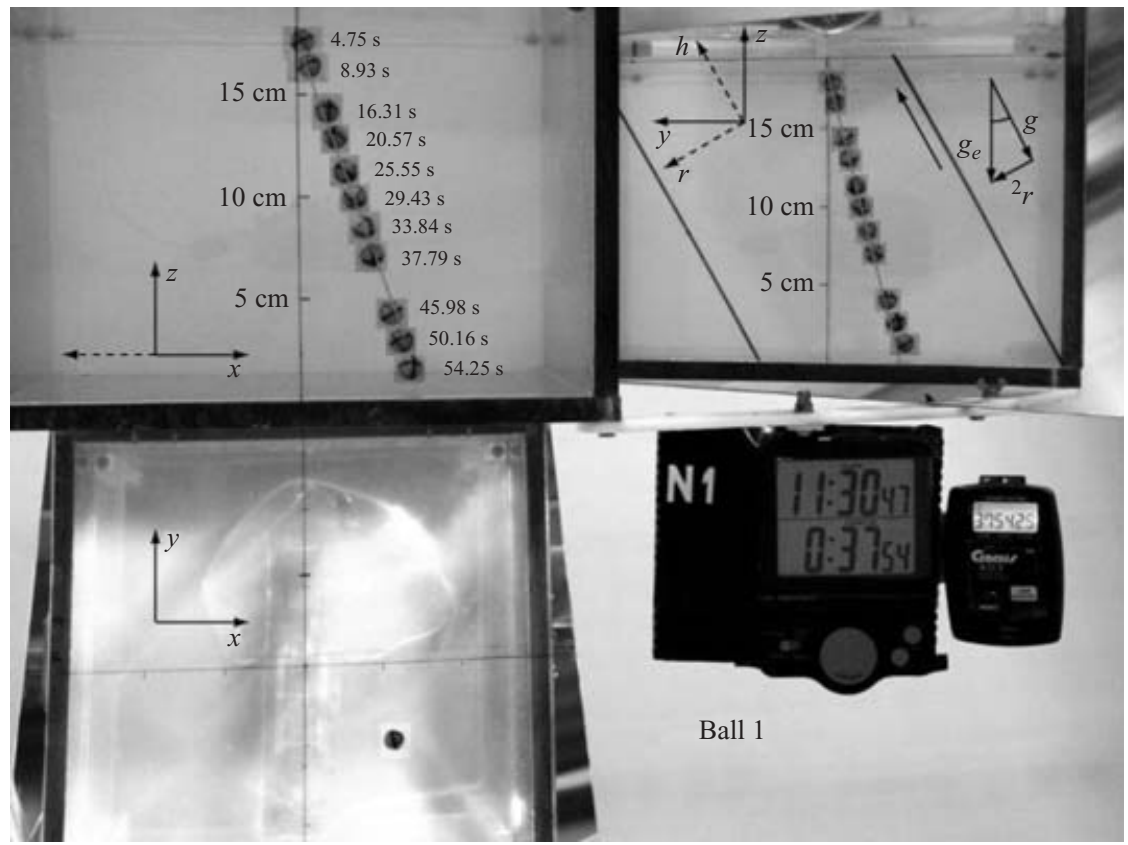

FIGURE 2. A photograph showing the progress of a typical experiment N1. Three views are captured simultaneously: front view (top left), side view (top right), and bottom view (lower left). The coordinate axes $(x, y, z)$ and $(r, \lambda, h)$ are shown by black arrows. Consecutive images are overlapped to reveal the trajectory of ball B1 with numbers to the right indicating time in seconds. In the bottom view, only the last image corresponding to $t=54.25 \mathrm{~s}$ is shown to avoid crowding. The three-dimensional coordinates of the ball $(x, y, z)$ were reconstructed from the measured positions in the front and side views and taking into account the depth of the image by using the perspective projection transformation.

to make corrections for the effects of variation of viscosity and thermal expansion (see the Appendix).

Below the wooden arm a digital $1600 \times 1200$ pixel camera was mounted to record the motion of the balls. Two mirrors were attached to the tank at an angle, one below it to provide a view from the bottom, and one on the right-hand side, providing a view from the side onto the motion in the tank (figure 2). A stop watch with $0.01 \mathrm{~s}$ resolution was also attached to the tank, to monitor the spin-up time, to record the time of release of the spheres, and to measure their time of descent. The spin-up time was $30 \mathrm{~min}$, and after a ball had reached the bottom, we waited approximately 2 to 3 min before releasing the next ball in order to allow transient motions to subside.

To record and analyse the motion of spheres two right Cartesian coordinate systems (as in Sheremet 2004) rotating with the centrifuge are introduced. The system $r, \lambda, h$ is associated with the centrifuge, where $r$ is the radius from the centre of the turntable, $\lambda$ is the azimuthal angle measured in the direction of rotation, and $h$, measured vertically, is the height above the laboratory floor. This system is convenient to analyse the forces acting on a moving sphere because $h$ is aligned with the axis of rotation $\Omega$. The system $(x, y, z)$ aligned with the tilted tank is convenient for measuring the position of a sphere inside the tank. The axes $(y, z)$ are in the same plane as $(r, h)$ but rotated inward through the angle $\alpha ; x$ points in the opposite direction compared with $\lambda$. Both coordinate systems are shown in figure 1 . By measuring the position of a ball in the front and side views and taking into account the depth of the image by 


$\begin{array}{clccc}\begin{array}{clc}R \\ \text { Experiments }\end{array} & \begin{array}{c}R \\ (\mathrm{~m})\end{array} & \begin{array}{c}\Omega \\ \left(\mathrm{rad} \mathrm{s}^{-1}\right)\end{array} & \begin{array}{c}\alpha \\ (\mathrm{deg} .)\end{array} & \begin{array}{c}T_{F} \\ \left({ }^{\circ} \mathrm{C}\right)\end{array} \\ \text { N1 } & 2.46 & 1.517 & 30 & 24.2 \\ \text { N2 } & 2.46 & 1.517 & 30 & 24.2 \\ \text { N3 } & 2.46 & 1.517 & 30 & 24.2 \\ \text { R1 } & 0 & 1.517 & 0 & 24.5 \\ \text { R2 } & 0 & 1.517 & 0 & 26.0 \\ \text { R3 } & 0 & 1.517 & 0 & 25.5 \\ \text { R4 } & 0 & 1.517 & 0 & 25.0 \\ \text { R5 } & 0 & 1.314 & 0 & 25.0 \\ \text { R6 } & 0 & 1.314 & 0 & 25.0 \\ \text { R7 } & 0 & 1.314 & 0 & 25.0 \\ \text { S1 } & 0 & 0 & 0 & 25.6 \\ \text { S2 } & 0 & 0 & 0 & 25.3 \\ \text { S3 } & 0 & 0 & 0 & 25.2 \\ \text { S4 } & 0 & 0 & 0 & 25.0\end{array}$

TABLE 1. Summary of experiments and parameters.

using the perspective projection transformation, the three-dimensional coordinates of the ball $(x, y, z)$ relative to the tank are reconstructed. The velocity components in the system $(r, \lambda, h)$ using the rotation transformation

$$
V_{\lambda}=-V_{x}, \quad V_{r}=\cos \alpha V_{y}-\sin \alpha V_{z}, \quad V_{h}=\sin \alpha V_{y}+\cos \alpha V_{z}
$$

are then obtained.

For experiments with the motion of the sphere along the axis of rotation (series $\mathrm{R}$ ) and with no rotation (series S), the tank was mounted at the centre of the rotating table and a similar experimental procedure was followed using the same nylon balls and salty water, which was saved and then used to refill the tank after it had been moved. All experimental parameters are summarized in tables 1 and 2.

\section{Taylor column formation and wall effects}

Proudman (1916) discovered analytically that in a geostrophically balanced flow, for sufficiently low Rossby numbers, the velocity field gradient along the axis of rotation is zero:

$$
(\boldsymbol{\Omega} \cdot \nabla) \boldsymbol{u}=0 .
$$

This result was experimentally confirmed by Taylor (1917) and is referred to as the Taylor-Proudman theorem. The laboratory experiments showed the formation of a Taylor column: fluid in the column aligned with the rotation axis moves with the body as a whole. If the boundaries of the tank are at a large distance from the sphere or at infinity in a theoretical consideration, the Taylor column will be gradually 'eroded' by viscous and inertial effects far away from the sphere. In this case a truncated Taylor column or a 'Taylor slug' (Maxworthy 1970) is observed. Affected by inertia, viscosity, direction of the motion, and interaction with the walls, the picture of the Taylor column formation is complicated. The only sure way of knowing would be to visualize the flow around the sphere with dye; this was not attempted in the experiments presented here. It is, however, important to have some idea of whether one might expect a Taylor column or slug, as the interaction of either of these will have effects on the way the spheres move within the tank. 


\begin{tabular}{|c|c|c|}
\hline Fluid: & $\begin{array}{l}15.79 \% \mathrm{NaCl} \text { aqueous solution } \\
\text { density of fluid }\end{array}$ & \\
\hline$\rho_{20}$ & density at $20^{\circ} \mathrm{C}$ & $1.11456 \mathrm{~g} \mathrm{~cm}^{-3}$ \\
\hline$\rho_{25}$ & density at $25^{\circ} \mathrm{C}$ & $1.11235 \mathrm{~g} \mathrm{~cm}^{-3}$ \\
\hline$\alpha_{F}=-\frac{1}{\rho}\left(\frac{\partial \rho}{\partial T}\right)$ & coefficient of thermal expansion at $25^{\circ} \mathrm{C}$ & $406 \times 10^{-6}{ }^{\circ} \mathrm{C}^{-1}$ \\
\hline 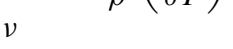 & kinematic viscosity of fluid & see $(\mathrm{A} 3)$ \\
\hline & kinematic viscosity at $25^{\circ} \mathrm{C}$ & $1.1028 \times 10^{-2} \mathrm{~cm}^{2} \mathrm{~s}^{-1}$ \\
\hline$\frac{1}{v}\left(\frac{\partial v}{\partial T}\right)$ & thermal coefficient of kinematic viscosity at $25^{\circ} \mathrm{C}$ & $-0.02238^{\circ} \mathrm{C}^{-1}$ \\
\hline$T_{F}$ & temperature of the fluid during each experiment & $24-26^{\circ} \mathrm{C}$ \\
\hline $\begin{array}{l}\text { Spheres: } \\
a \\
\rho+\Delta \rho\end{array}$ & $\begin{array}{l}\text { Nylon } 6 / 6 \text { balls } \\
\text { radius of the ball } \\
\text { density of the ball }\end{array}$ & $0.635 \mathrm{~cm}(1 / 4 \mathrm{in})$ \\
\hline $\begin{array}{l}\Delta \rho \\
\Delta \rho_{25}\end{array}$ & $\begin{array}{l}\text { density difference between sphere and fluid } \\
\Delta \rho \text { referenced to } 25^{\circ} \mathrm{C}\end{array}$ & $\begin{array}{l}\sim 10^{-3} \mathrm{~g} \mathrm{~cm}^{-3} \\
\text { see (A } 4)\end{array}$ \\
\hline $\begin{array}{l}\alpha_{N}=-\frac{1}{\rho}\left(\frac{\partial \rho}{\partial T}\right) \\
\text { Centrifuge: }\end{array}$ & coefficient of cubical thermal expansion at $20^{\circ} \mathrm{C}$ & $265 \times 10^{-6}{ }^{\circ} \mathrm{C}^{-1}$ \\
\hline$R$ & $\begin{array}{l}\text { distance from the axis of rotation } \\
\text { to the center of the experimental compartment } \\
\text { of the tank filled with fluid }\end{array}$ & 2.46 or $0 \mathrm{~m}$ \\
\hline$\Omega$ & angular rate of rotation & 1.517 or $1.314 \mathrm{rad} \mathrm{s}^{-1}$ \\
\hline$\alpha$ & tilt of the tank & $30^{\circ}$ or $0^{\circ}$ \\
\hline$g$ & gravitational acceleration & $980.3 \mathrm{~cm}^{2} \mathrm{~s}^{-1}$ \\
\hline
\end{tabular}

TABLE 2. Key experimental parameters and material properties. The same nylon balls and salty water was used in all experiments.

\subsection{Motion along the axis of rotation}

For coaxial motion of the particle with speed $V_{\|}$, Taylor (1922) observed that the Rossby number $R o_{\|}=V_{\|} / 2 \Omega a$, where $a$ is the radius of the sphere, must be less than a critical value $1 / 2 \pi$ for there to be a columnar structure accompanying the sphere. Other experiments have suggested critical values as high as 0.35 (Pritchard 1969) for substantial Taylor column formation. In our tilted experiments the Rossby number was typically between about 0.07 and 0.3 for motion parallel to the axis of rotation. These numbers suggest that there may or may not be a column depending on which criterion you believe. For motion at negligibly small Rossby numbers, the vertical extent of the Taylor column is determined by the fluid viscosity. The theory (Moore \& Saffman 1969) predicts that a truncated Taylor column, or 'Taylor slug', will extend a characteristic distance $a \mathscr{T}$ up- and downstream of a sphere of radius $a$, where $\mathscr{T}=\Omega a^{2} / v$ is the Taylor number. Maxworthy (1970), however, found experimentally that the column is typically an order of magnitude smaller than that which was also reported for the axial motion of drops by Bush, Stone \& Bloxham (1995). Similarly, the numerical results of Tanzosh \& Stone (1994) for a sphere translating at $R o_{\|}=0$ indicate a blocked region of length $0.116 a \mathscr{T}$. Based on these findings, a Taylor slug extending approximately $3 \mathrm{~cm}$ fore and aft of the sphere may be expected.

\subsection{Motion perpendicular to the axis of rotation}

For transverse motion the criteria are less well understood. Here the sphere simply translates through the fluid, perpendicular to the axis of rotation, taking with it a cylindrical body of fluid, extending above and below. External fluid will stream past 
this body of fluid as though it were a solid body. This is unlike the coaxial motion, where the sphere is actually trying to move up and down in the fluid, and thus to defy the Taylor-Proudman theorem. The Hide criterion for Taylor column formation states that such a column may only form if $h / D>R o_{\perp}$, where $h$ is the vertical length of the particle (its diameter in the case of a sphere) and $D$ the depth of the fluid layer; $R o_{\perp}$ is defined to be $V_{\perp} / 2 a \Omega$ in the case of a sphere. In our setup $h / D=0.0635$ where typically $R o_{\perp}=0.071$, so again the experiments presented here are on the borderline. Experiments by Hide \& Ibbetson (1966), however, have shown that the Taylor columns only form for transverse motion when $h / D>0.5 R o_{\perp}$ in which case our numbers would predict no column. Furthermore, Hide, Ibbetson \& Lighthill (1968) observed that the Taylor column trails in the downstream direction at a small angle $\psi=\arctan \left(K R o_{\perp}\right)$ proportional to Rossby number, with the coefficient $K=(1.54 \pm 0.04)$ for a sphere. This result was explained based on the direction of group speed of the inertial (gyroscopic) waves.

\subsection{Wall effects}

The centre-rotation experiments show some evidence of wall effects on the velocity of descent of the sphere. In all the plots of the vertical position versus time, it can be seen that the sphere first accelerates slightly after it exits the hole in the upper lid and then slows down as it approaches the bottom. This can be explained by a Taylor slug interacting with the lid as the ball is released and gradually losing its efficiency in slowing the ball down as it moves away from the wall. As the slug starts interacting with the bottom boundary it again slows the vertical motion of the sphere. An analysis of the increasing drag, when the Taylor column interacts with top or bottom boundaries, can be found in Moore \& Saffman (1968), Maxworthy (1968), Hocking, Moore \& Walton (1979), and Tanzosh \& Stone (1994).

For the experiments presented here it will be assumed that a small Taylor slug forms around the spheres, but not a full Taylor column. This assumption is supported by the fact that Stewartson's theory, to which the results are compared, was developed for the unbounded case, and it agrees reasonably well with our experimental results, as will be shown.

\section{Forces on a spherical particle moving in a rotating fluid}

For a particle of mass $m$ moving at the speed $V$ relative to a reference frame rotating at the angular velocity $\Omega$ Newton's law is

$$
m \frac{\mathrm{d} \boldsymbol{V}}{\mathrm{d} t}=-m \cdot 2 \boldsymbol{\Omega} \times \boldsymbol{V}+\boldsymbol{F}_{S}+m \boldsymbol{g}+m \Omega^{2} \boldsymbol{r} .
$$

The relative acceleration term on the left-hand side is balanced on the right-hand side (in order) by the Coriolis force $\mathbf{F}_{C}$, acting to the right of the moving particle if viewed from the end of the $\boldsymbol{\Omega}$ vector, the pressure and viscous stress forces $\boldsymbol{F}_{S}$ applied at the surface of the particle, the gravitational force and the centrifugal force proportional to the distance $r$ from the axis of rotation and directed radially; $\boldsymbol{r}$ is perpendicular to the axis of rotation.

To find $\boldsymbol{F}_{S}$ the Navier-Stokes equations governing the flow around the particle are considered:

and the continuity equation

$$
\frac{\mathrm{d} \boldsymbol{u}}{\mathrm{d} t}+2 \boldsymbol{\Omega} \times \boldsymbol{u}=-\frac{1}{\rho} \nabla p+v \nabla^{2} \boldsymbol{u}+\boldsymbol{g}+\Omega^{2} \boldsymbol{r},
$$

$$
\nabla \cdot \boldsymbol{u}=0
$$


where $\boldsymbol{u}$ is the fluid velocity vector, $p$ is pressure, $\mathrm{d} / \mathrm{d} t$ is the substantial time derivative, and $\rho$ is the density and $v$ the viscosity of water. At the surface of the particle the fluid velocity is equal to that of the particle:

$$
\left.\boldsymbol{u}\right|_{S}=\boldsymbol{V}+\boldsymbol{\omega}_{B} \times \text { an, }
$$

and at the walls of the tank rotating with the reference frame $\boldsymbol{u}$ vanishes. Here $\boldsymbol{\omega}_{B}$ represents possible angular velocity of the ball and $\mathbf{n}$ is the outer normal to its surface. Moore \& Saffman (1969) showed that the effect of the free rotation on the drag can be of the order of Rossby number.

The gravitational and centrifugal forces can be combined and the 'effective' free-fall acceleration is introduced:

$$
\boldsymbol{g}_{e}=\boldsymbol{g}+\Omega^{2} \boldsymbol{r}
$$

which depends on the distance from the axis of rotation $r$ (see Fletcher 1972 for a discussion and experimental validation of the apparent field of gravity in a rotating fluid system). Since the size of our experimental tank is much smaller than the distance from the axis of rotation, $r$ is replaced in (4.5) by a constant value $R=2.46 \mathrm{~m}$ representing the distance from the axis of rotation to the centre of mass of the fluid in the tank.

The pressure can be divided into the dynamic $\left(p_{d}\right)$ and hydrostatic components

$$
p=p_{d}+\rho\left(g\left(h_{0}-h\right)+\frac{1}{2} \Omega^{2} r^{2}\right),
$$

where the reference constant $h_{0}$ represents the height the free surface of fluid at the centre of rotation. The hydrostatic pressure integrated over the surface of the particle results in the Archimedes force, which, combined with the gravitational and centrifugal forces, produces the buoyancy force

$$
\boldsymbol{F}_{B}=\left(m-m_{0}\right) \boldsymbol{g}_{e}=\frac{4}{3} \pi a^{3} \Delta \rho \boldsymbol{g}_{e} .
$$

Here $m_{0}$ is the mass of fluid displaced by the particle, $\Delta \rho$ is the density difference between the material of the particle and fluid, and $a$ is the radius of a spherical particle, $a \ll R$.

The dynamic pressure and viscous stress integrated over the surface of the particle result in the hydrodynamic force which is determined by the flow of fluid around a moving particle:

$$
\boldsymbol{F}_{H}=-\int_{S} p_{d} \boldsymbol{n} \mathrm{d} S+\int_{S} \boldsymbol{\tau} \cdot \boldsymbol{n} \mathrm{d} S,
$$

where $\boldsymbol{n}$ is the outer normal to the surface element $\mathrm{d} S$ and $\boldsymbol{\tau}$ is the viscous stress tensor. The hydrodynamic force, in turn, can be decomposed into the $\operatorname{drag} \boldsymbol{F}_{D}$ pointing in the opposite direction to the particle velocity and lift $\boldsymbol{F}_{L}$ perpendicular to the velocity, $\boldsymbol{F}_{H}=\boldsymbol{F}_{D}+\boldsymbol{F}_{L}$. Thus, if a particle moves at a uniform speed (which was approximately true for the portion of trajectory away from the boundaries), the buoyancy, Coriolis, lift, and drag forces will be in balance:

$$
0=\boldsymbol{F}_{B}+\boldsymbol{F}_{C}+\boldsymbol{F}_{D}+\boldsymbol{F}_{L} .
$$

Figures $3(a)$ and $3(b)$ illustrate the force balance in the plane perpendicular to the axis of rotation $\boldsymbol{\Omega}$ and along the axis of rotation respectively.

In a rapidly rotating fluid it is very challenging to calculate the hydrodynamic force $\boldsymbol{F}_{H}$ because of radiation of inertial waves and development of a Taylor column around the particle. Two non-dimensional parameters play an important role here: the Rossby number $R o=V / 2 \Omega a$ is the ratio of nonlinear and Coriolis accelerations; 
(a)

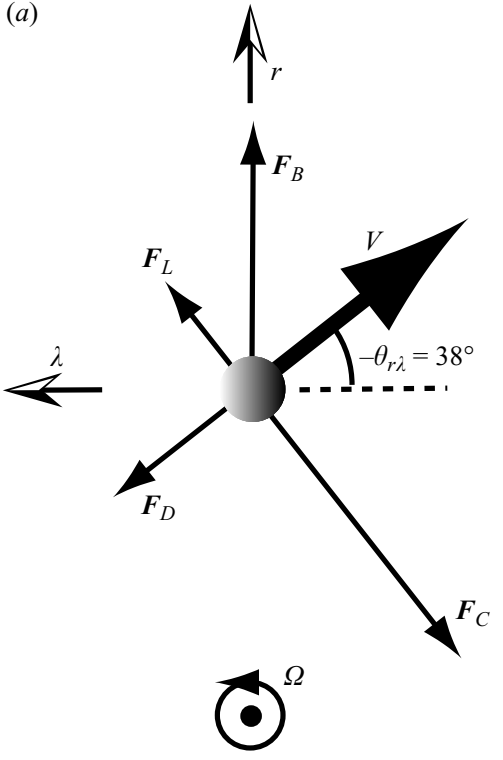

(b)

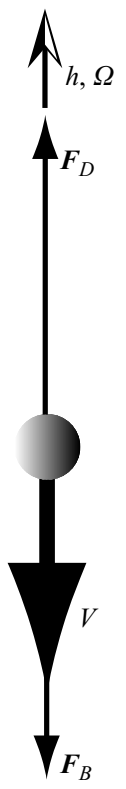

FiguRE 3. The balance of forces acting on a sphere: $(a)$ moving in a plane perpendicular to the axis of rotation $\boldsymbol{\Omega}$ (the rotation is counterclockwise); $(b)$ moving along the axis of rotation. The scales of the vectors and their orientations in $(a)$ are in accordance with (5.2), (5.3) and (5.4).

the Taylor number $\mathscr{T}=\Omega a^{2} / v$ is the square of the particle dimension $a$ over the Ekman thickness $h_{E}=\sqrt{v / \Omega}$. Also, the ratio $O(a \mathscr{T} / H)$, where $H$ is the height of the container, characterizes the interaction of the Taylor column with the walls if the fluid is bounded. Often, it is more convenient to use the Reynolds number $R e=2 \mathrm{Va} / \mathrm{v}=4 R o \mathscr{T}$. A comprehensive survey of particle motion in rotating fluids can be found in Bush, Stone \& Tanzosh (1994). Our primary interest stems from the geophysical applications and, hence, lies in the regime with $R o \ll 1$ and $\mathscr{T} \gg 1$. In the tilted tank experiments $\mathscr{T}$ equalled approximately 48 and $R o$ (based on the component $V_{h}$ ) ranged from 0.07 to 0.31 .

\section{Stewartson's theory}

Most existing theoretical calculations of hydrodynamic forces for particles moving through rotating fluids neglect the inertial terms for $R o \ll 1$ and are, therefore, linear. Special note is given to the work of Stewartson (1953) who, using a Laplace transform, solved a linear inviscid problem of an ellipsoid (with a sphere being a special case) impulsively started from rest in an infinite rotating fluid. He calculated the pressure distribution on the surface after a long time had passed and found the drag and lift on a uniformly moving ellipsoid due to the generation of inertial waves and development of a Taylor column.

Stewartson (1953) found that in motion along the axis of rotation with velocity $V_{h}$, a sphere experiences a drag

$$
F_{D h}=-\frac{16}{3} \rho a^{3} \Omega V_{h} .
$$

Physically this means that the generated inertial waves interfere to produce the developing Taylor column in front of and behind the moving sphere. In front of the 
sphere, where fluid tends to diverge, an anticyclonic swirling motion develops with typical velocities $(\Omega a)$ and correspondingly positive pressure anomaly. Behind the sphere the swirling motion is cyclonic and the pressure anomaly is negative. This produces the drag on the sphere. The extent of the developing Taylor column grows linearly with time: the work done by the drag force in Stewartson's theory (or the potential energy released as the sphere descends in a gravity field) goes into ever growing kinetic energy of the swirling motion. When moving perpendicular to the axis of rotation with, for example, velocity $V_{r}$ a sphere experiences both drag

$$
F_{D r}=-\frac{4}{3} \pi a^{3} \rho \frac{4 \pi}{16+\pi^{2}} 2 \Omega V_{r}
$$

and lift

$$
F_{L \lambda}=\frac{4}{3} \pi a^{3} \rho \frac{\pi^{2}}{16+\pi^{2}} 2 \Omega V_{r},
$$

acting to the left if viewed from the end of $\boldsymbol{\Omega}$. These were also determined by Stewartson (1953) and here the non-tilted system of coordinates $(r, \lambda, h)$ was used to indicate directions.

For small density difference between the particle and fluid, $\Delta \rho \ll \rho$, the Coriolis force

$$
F_{C \lambda}=-\frac{4}{3} \pi a^{3}(\rho+\Delta \rho) 2 \Omega V_{r},
$$

acting to the right but stronger than the lift force, can be added to a single matrix equation. It relates the velocity of an ellipsoid and the sum of drag, lift, and Coriolis forces acting on it (since they are linearly dependent on velocity):

$$
\left(\begin{array}{c}
F_{r} \\
F_{\lambda} \\
F_{h}
\end{array}\right)=-\frac{16}{3} \rho \Omega a^{3}\left[\begin{array}{ccc}
\frac{2 \pi^{2}}{16+\pi^{2}} & -\frac{8 \pi}{16+\pi^{2}} & 0 \\
\frac{8 \pi}{16+\pi^{2}} & \frac{2 \pi^{2}}{16+\pi^{2}} & 0 \\
0 & 0 & 1
\end{array}\right]\left(\begin{array}{c}
V_{r} \\
V_{\lambda} \\
V_{h}
\end{array}\right) .
$$

Here it is appropriate to note that the fluid flow compensating displacement of the sphere also experiences a Coriolis force, which, in turn, results in pressure anomalies causing the lift force.

In order to obtain the velocity of a sphere resulting from the applied buoyancy force $\boldsymbol{F}_{B}(4.7)$, this matrix is inverted to obtain

$$
\left(\begin{array}{l}
V_{r} \\
V_{\lambda} \\
V_{h}
\end{array}\right)=\frac{\pi}{4 \Omega} \frac{\Delta \rho}{\rho}\left[\begin{array}{ccc}
1 / 2 & 2 / \pi & 0 \\
-2 / \pi & 1 / 2 & 0 \\
0 & 0 & 1
\end{array}\right]\left(\begin{array}{l}
g_{e r} \\
g_{e \lambda} \\
g_{e h}
\end{array}\right) \text {. }
$$

In our case the 'effective' gravitational acceleration vector projected onto $(r, \lambda, h)$ has components $\left(g_{e r}, g_{e \lambda}, g_{e h}\right)=\left(\Omega^{2} R, 0,-g\right)=(g \tan \alpha, 0,-g)$. Multiplication by the matrix (5.6) gives the velocity vector:

$$
\left(\begin{array}{c}
V_{r} \\
V_{\lambda} \\
V_{h}
\end{array}\right)=\frac{\pi}{4 \Omega} \frac{\Delta \rho}{\rho}\left(\begin{array}{c}
\frac{1}{2} \Omega^{2} R \\
-(2 / \pi) \Omega^{2} R \\
-g
\end{array}\right)=\frac{\pi}{4 \Omega} \frac{\Delta \rho}{\rho} g\left(\begin{array}{c}
\frac{1}{2} \tan \alpha \\
-(2 / \pi) \tan \alpha \\
-1
\end{array}\right) .
$$

As a sphere sinks, $V_{h}<0$, it drifts away from the centre, $V_{r}>0$, and also moves azimuthally, lagging the rotation of the centrifuge, $V_{\lambda}<0$. It is convenient to introduce 
the angles between the trajectory and the coordinate axes:

$$
\begin{gathered}
\theta_{r h}=\arctan \left(V_{r} / V_{h}\right)=-\arctan \left(\frac{1}{2} \tan \alpha\right), \\
\theta_{\lambda h}=\arctan \left(V_{\lambda} / V_{h}\right)=\arctan ((2 / \pi) \tan \alpha), \\
\theta_{r \lambda}=\arctan \left(V_{r} / V_{\lambda}\right)=-\arctan \left(\frac{1}{4} \pi\right)=-38.1460^{\circ} .
\end{gathered}
$$

Thus, in the linear approximation the trajectory is independent of the particle's density anomaly. For $\alpha=30^{\circ},-\theta_{r h}=16.10^{\circ}$ and $\theta_{\lambda h}=20.18^{\circ}$. Furthermore, in the plane perpendicular to the axis of rotation, the particle (for $\Delta \rho / \rho \ll 1$ ) moves at the fixed (independent of $\alpha$ ) angle $90^{\circ}-38.1460^{\circ}=51.8540^{\circ}$ clockwise from the direction of the centrifugal acceleration (figure $3 a$ ).

It is interesting to note that the prediction for the trajectory of a solid sphere according to Stewartson's inviscid theory is identical to that according to the viscous theory of Loper (2001) for a spherical fluid parcel with the Gaussian distribution of density anomaly. Specifically, the force-velocity matrix relations (5.6) and Loper's equation (8.9) are same. If the total mass anomaly is assumed to be the same in both cases, the solid particle will move $2 \sqrt{2}$ times faster. Such a surprising agreement between the two different theories can be interpreted in the following way. Essentially, the hydrodynamic reaction of the fluid on a moving particle or a parcel is determined by the (approximately linear) flow around a truncated Taylor column, which is common to both Stewartson's and Loper's work. However, the establishment of the Taylor column is limited by the time-dependent effects in the case of Stewartson and by weak viscous effects in the case of Loper. Note also that Sheremet (2004) analysed trajectories of fluid blobs in a similar experimental setup and found good agreement with Stewartson's prediction if the deformation of a blob is taken into account and the blob is modeled as an ellipsoid.

\section{Experimental results}

Several series of experiments were conducted (table 1). The tilted tank configuration series (N1, N2, and N3) allowed the three-dimensional trajectories of falling balls to be analysed and information about the relative magnitudes and directions of drag and lift forces to be inferred. In the centre-rotation experiments (R1, R2, R3, and R4) the same angular velocity as the N-series was used, while in R5, R6, and R7 the angular velocity was reduced by the factor of $\cos \left(30^{\circ}\right)=0.8660$ corresponding to the projection of $\boldsymbol{\Omega}$ on the tilted axis $z$ in the $\mathrm{N}$ series. This allowed the role of variation of the angular velocity and the tilt angle on the magnitude of the velocities to be assessed. Finally, the non-rotating experiments (S1, S2, S3, and S4) were used to estimate the density of the balls from their terminal velocities. The intention was to repeat each experiment three times in order to check the reliability of measurements. However, occasionally outliers occurred primarily due to appearance of tiny air bubbles on the surface of the balls; therefore, some experiments were repeated four times.

\subsection{Tilted tank experiments}

The progress of a typical tilted tank experiment N1 is shown in figure 2. Three views are captured simultaneously: front view (top left), side view (top right), and bottom view (lower left) using two mirrors. The coordinate axes $(x, y, z)$ and $(r, \lambda, h)$ are shown by black arrows. Consecutive images are overlapped to reveal the trajectory of the ball $\mathrm{B} 1$ with numbers to the right indicating time in seconds. In the bottom view, only the last image corresponding to $t=54.25 \mathrm{~s}$ is shown to avoid crowding. 

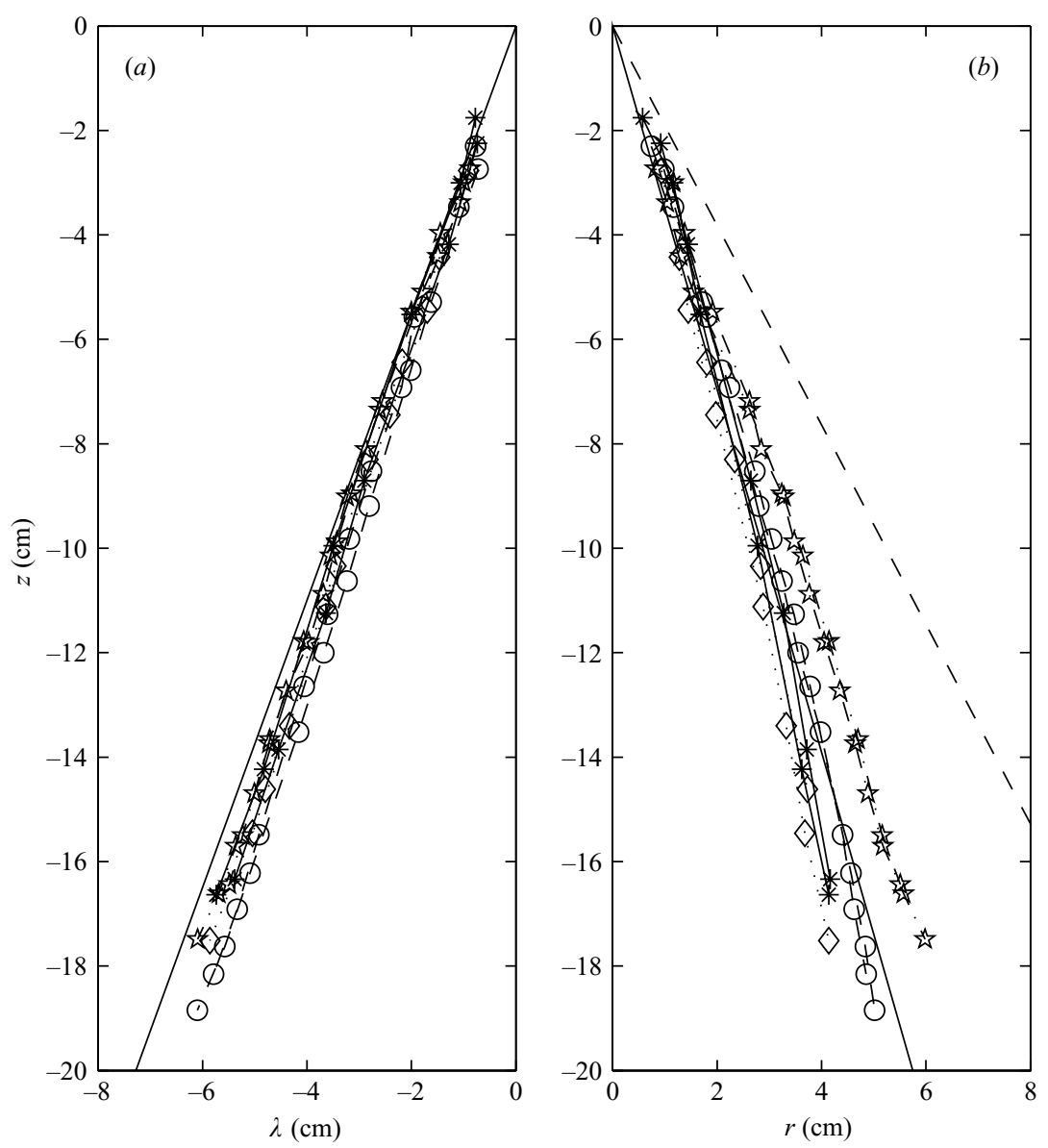

FIgURE 4. (a) The azimuthal trajectories and $(b)$ the radial trajectories of balls B1, B2, B5 and B6 in experiments N1 and N2. The measurements of the location of the balls are marked for ball B1 by circles $(O)$ connected by a dashed line; B2, diamonds $(\diamond)$ connected by a dotted line; B5, pentagram ( 2 ) connected by a dashed-dotted line; B6, stars (*) connected by a solid line. The solid line without markers shows the angle predicted by linear theory and in both directions the angle agrees reasonably well with the paths taken by the spheres plotted using the symbols. Note that the axis of rotation is parallel to the vertical axis in the plots. The dashed line indicates the effective gravity $\boldsymbol{g}_{e}$.

The three-dimensional coordinates of the ball $(x, y, z)$ were reconstructed from the measured positions in the front and side views by taking into account the depth of the image using the perspective projection transformation. Then using (2.2) the positions and velocities relative to $(r, \lambda, h)$ were reconstructed for further analysis.

The reconstructed trajectories of all four balls in experiments N1 and N2 are shown in figure 4. It is seen that the trajectories of individual balls are reproduced well from one experiment to another. The prediction of (5.7) is shown by a solid line and is in good quantitative agreement. Note that the scatter of trajectories is larger for the radial displacement than for the azimuthal. The descent velocity of a ball was fairly constant except near the top and bottom (within approximately $3 \mathrm{~cm}$ ) due to the interaction between the wall and the Taylor slug. Plotting a coordinate as a function of time and then fitting a straight line for the middle part of the tank 


\begin{tabular}{cccccccc}
\hline \multirow{2}{*}{ Ball } & Exp. & $V_{r}$ & $V_{\lambda}$ & $V_{h}$ & $R e_{\|}$ & $R o_{\|}$ & $\omega_{B} / \Omega$ \\
B1 & N1 & 0.0846 & -0.1022 & -0.3252 & 37.45 & 0.1688 & -0.1035 \\
& N2 & 0.0834 & -0.1071 & -0.3283 & 37.81 & 0.1704 & -0.1035 \\
& N3 & 0.0374 & -0.0388 & -0.1301 & 14.98 & 0.0675 & -0.0122 \\
B2 & N1 & 0.1119 & -0.1698 & -0.5084 & 58.55 & 0.2639 & -0.1972 \\
& N2 & 0.1180 & -0.1588 & -0.4873 & 56.12 & 0.2529 & -0.1883 \\
& N3 & 0.1129 & -0.1480 & -0.4571 & 52.64 & 0.2373 & -0.1726 \\
B5 & N1 & 0.0731 & -0.0710 & -0.2083 & 23.99 & 0.1081 & -0.0406 \\
& N2 & 0.0719 & -0.0748 & -0.2208 & 25.43 & 0.1146 & -0.0499 \\
& N3 & 0.0623 & -0.0579 & -0.1922 & 22.13 & 0.0998 & -0.0414 \\
B6 & N1 & 0.1256 & -0.1807 & -0.5404 & 62.23 & 0.2805 & -0.2180 \\
& N2 & 0.1363 & -0.1930 & -0.5826 & 67.09 & 0.3024 & -0.2071 \\
& N3 & 0.1345 & -0.1704 & -0.5165 & 59.48 & 0.2681 & -0.1801
\end{tabular}

TABLE 3. The velocity results from the tilted tank experiments: the radial component, $V_{r}$, the azimuthal component, $V_{\lambda}$, the vertical component, $V_{h}$, parallel to the axis of rotation; the units are $\mathrm{cm} \mathrm{s}^{-1}$. The next two columns specify the Reynolds number $R e_{\|}=2\left|V_{h}\right| a / v_{25}$ and the Rossby number $R o_{\|}=\left|V_{h}\right| / 2 \Omega a$ for axial motion of each ball in the respective experiment. The last column lists the scaled spinning of the ball $\omega_{B}$ relative to the fluid. The negative sign means anticyclonic rotation (opposite to the centrifuge). The Taylor number $\mathscr{T}=\Omega a^{2} / \nu_{25}=55.47$ is fixed in the $\mathrm{N}$-series.

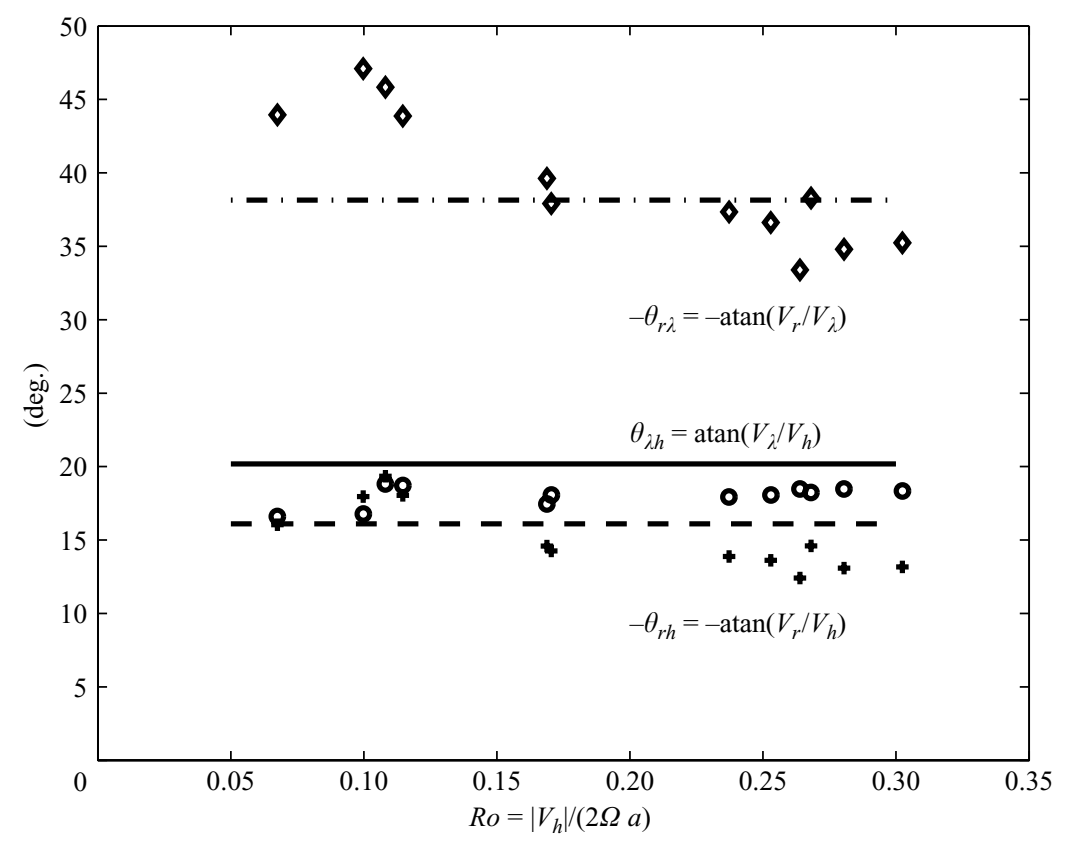

FIGURE 5. The trajectory angles $-\theta_{r \lambda}(\diamond), \theta_{\lambda h}(\bigcirc),-\theta_{r h}(+)$ for the tilted tank experiments $\mathrm{N}$ versus the Rossby number based on the axial velocity $V_{h}$. The horizontal lines show the corresponding values predicted by (5.8), (5.9), and (5.10).

$(5 \mathrm{~cm}<z<15 \mathrm{~cm})$ gives a set of interior velocities for each ball and experiment that are listed in table 3. Also listed are the Rossby and Reynolds numbers for the motion in the axial and perpendicular directions. Due to natural scatter in the density of each ball, which will be discussed later, the Rossby number based on the axial velocity $R o_{\|}=-V_{h} /(2 \Omega a)$ ranged from 0.07 to 0.3 . In figure 5 the angles of propagation based 
on the interior velocities as a function of $R o_{\|}$are plotted. The experimental data are in good quantitative agreement with Stewartson's prediction (5.8), (5.9), (5.10) shown by straight lines. The most sensitive to variation in the Rossby number is the angle $-\theta_{r \lambda}$, which describes the motion in a plane perpendicular to the axis of rotation; it shows some decrease from $45^{\circ}$ to $35^{\circ}$ as $R o_{\|}$increases. The agreement is best for $R o_{\|} \simeq 0.2$ and not for infinitesimal $R o_{\|}$.

Finally, in figure 2 one can notice a slow anticyclonic (opposite to the centrifuge) spinning of the ball by observing the change in orientation of coloured markers on its surface as the ball descends. One full rotation is accomplished in approximately $40 \mathrm{~s}$, from position at $8.93 \mathrm{~s}$ to $50.16 \mathrm{~s}$. The spinning rate $\omega_{B}$ is found to increase with the Rossby number $R o_{\|}$. The ratio $\omega_{B} / \Omega$ is listed in the last column of table 3 . These are values averaged for motion through the interior of tank. Not enough images were available to quantify any changes in the spinning rate near the boundaries.

The spinning of a sphere moving along the axis of a rotating fluid was noted by Taylor (1922). He observed (viewed from the non-rotating laboratory reference frame) that, if the motionless ball, held by a silk thread, and fluid were first both rotating as a solid body with the glass cylinder and then the ball was allowed to move, the ball stopped rotating as soon as it started moving along the axis. Physically, this can be explained by the conservation of absolute angular momentum: the fluid that starts to flow around the surface of the ball diverges from its pole and thus preserves zero azimuthal velocity. The zero absolute angular momentum is transferred to the ball, which stops rotating. In the reference frame rotating with the tank, the ball acquires anticyclonic spin $\omega_{B}=-\Omega$. Taylor found that this effect becomes pronounced when $R o_{\|}>1 /(2 \pi)$. For smaller $R o_{\|}$the Taylor column forms fore and aft with accompanying fluid circulating in the opposite sense on both sides; therefore, this effect is weakened. In the linear case, in particular when the Taylor column starts to interact equally with both the solid top and bottom lids of the container, the moving ball should not be spinning relative to the fluid (Moore \& Saffman 1968).

\subsection{Centre-rotation experiments}

The drag on a sphere in a rotating fluid generally increases with increasing rotation rate resulting in a smaller descent velocity. In a certain range of Reynolds numbers and for small rotation rates, the rotation can stabilize the time-dependent flow and, on the contrary, reduce the drag (Maxworthy 1970). In order to estimate the effects of tilting on the speed of descent, a set of experiments was carried out with the same tank as in the tilted case, but placed at the centre of the rotating table. In the first set of experiments (R1, R2, R3 and R4) the rotation rate was kept the same as in the tilted experiments $\left(\Omega=1.517 \mathrm{~s}^{-1}\right)$. However, in a second run of experiments (R5, R6 and R7) the rate of rotation was lowered slightly so that it would correspond to the 'vertical' component (the axis $z$ in figure 2 i.e., along which effective gravity acts) of the rotation in the tilted case $\left(\Omega=1.517 \cos \left(30^{\circ}\right)=1.314 \mathrm{~s}^{-1}\right)$. The mean descent velocities in the interior of the tank $(5 \mathrm{~cm}<z<15 \mathrm{~cm})$ for each ball are listed in tables $4(a)$ and $4(b)$.

The data show that the descent velocity in the slower rotating experiments (R5, R6 and R7) is consistently larger than that in the faster rotating experiments R1R4. This illustrates that the Taylor-Proudman theorem becomes more effective as rotation increases in our case. The larger variability in experiments R1-R4 is due to temperature fluctuations between the experiments. 
(a)

\begin{tabular}{ccc}
\hline Ball & Exp. & $V_{h}$ \\
B1 & R1 & -0.2314 \\
& R2 & -0.3529 \\
& R3 & -0.3053 \\
& R4 & -0.2666 \\
B2 & R1 & -0.2292 \\
& R2 & -0.5356 \\
& R3 & -0.4490 \\
& R4 & -0.3996 \\
B5 & R1 & -0.1731 \\
& R2 & -0.2495 \\
& R3 & -0.2312 \\
& R4 & -0.1967 \\
B6 & R1 & -0.4168 \\
& R2 & -0.6417 \\
& R3 & -0.5838 \\
& R4 & -0.5183
\end{tabular}

$(b)$

\begin{tabular}{ccc}
\hline Ball & Exp. & $V_{h}$ \\
B1 & R5 & -0.3372 \\
& R6 & -0.3309 \\
& R7 & -0.3485 \\
& & \\
B2 & R5 & -0.5199 \\
& R6 & -0.5396 \\
& R7 & -0.5382 \\
& & \\
B5 & R5 & -0.2412 \\
& R6 & -0.2313 \\
& R7 & -0.2359 \\
& & \\
B6 & R5 & -0.7433 \\
& R6 & -0.7112 \\
& R7 & -0.7210
\end{tabular}

(c)

\begin{tabular}{ccc}
\hline Ball & Exp. & $V_{h}$ \\
B1 & S1 & -1.2647 \\
& S2 & -1.2458 \\
& S3 & -0.9469 \\
& S4 & -1.1845 \\
B2 & S1 & -1.6173 \\
& S2 & -1.5237 \\
& S3 & -1.4638 \\
& S4 & -1.4939 \\
B5 & S1 & -1.0645 \\
& S2 & -0.9941 \\
& S3 & -0.9705 \\
& S4 & -0.9365 \\
B6 & S1 & X \\
& S2 & -1.7061 \\
& S3 & -1.6967 \\
& S4 & -1.7147
\end{tabular}

TABLE 4. The velocity of descent $\left(\mathrm{cm} \mathrm{s}^{-1}\right)$ for each ball in $(a)$ experiments R1, R2, R3, and R4 at a rotation rate of $\Omega=1.517 \mathrm{~s}^{-1},(b) \mathrm{R} 5, \mathrm{R} 6$, and R7 at a rotation rate of $\Omega=1.314 \mathrm{~s}^{-1}$, and $(c)$ the non-rotating experiments $\mathrm{S} 1, \mathrm{~S} 2, \mathrm{~S} 3$, and S4. The datum for B6-S1 is missing.

\section{Drag in rotating fluid}

It has already been seen that in the tilted tank experiments Stewartson's theory predicts the angles of propagation of the balls very well, which, of course, should be independent of $\Delta \rho$ according to (5.8), (5.9), and (5.10). In order to see how well the theory predicts the magnitude of the velocity, a magnitude coefficient is introduced, defined as the ratio of the observed drag to that of Stewartson:

$$
A(R o, \mathscr{T})=\left(\frac{\pi}{4 \Omega} \frac{\Delta \rho}{\rho} g\right) /\left(-V_{h}\right)
$$

where $V_{h}$ is the vertical velocity component along the axis of rotation. The value of $A$ might weakly depend on Rossby and Taylor numbers for $R o \ll 1$ and $\mathscr{T} \gg 1$. The density anomaly $\Delta \rho$ estimates were obtained using the terminal descent velocity method which can be found in the Appendix. The descent velocity is shown in figure 6 . If Stewartson's prediction for the magnitude of the drag for given axial velocity is correct, then $A$ should be unity. The magnitude coefficient can be expressed in terms of the standard drag coefficient and Rossby number: $A=(3 \pi / 16) C_{D} R o$. Correcting the estimates of $\Delta \rho_{25}$ from the non-rotating $\mathrm{S}$ experiments for the temperatures observed during the rotating experiments $\mathrm{N}$ and $\mathrm{R}, A$ was calculated and is shown in figure $7(a)$. It is seen that the magnitude coefficient is scattered between 1 and 1.6, excepting the outliers. This means that the balls experience larger drag than predicted by the Stewartson's theory and sink somewhat slower.

It is interesting to make a comparison with laboratory experiments of Maxworthy (1970, figure 6). He measured the drag on a sphere moving along the axis of rotation in the range $23.3 \leqslant \mathscr{T} \leqslant 147,0.01 \leqslant R o \leqslant 1.27$ (in his notation $N=R o^{-1}$ ). $\mathrm{He}$ found that in the limit $R o \ll 1$ and $\mathscr{T} \gg 1$ the drag coefficient asymptotes to 


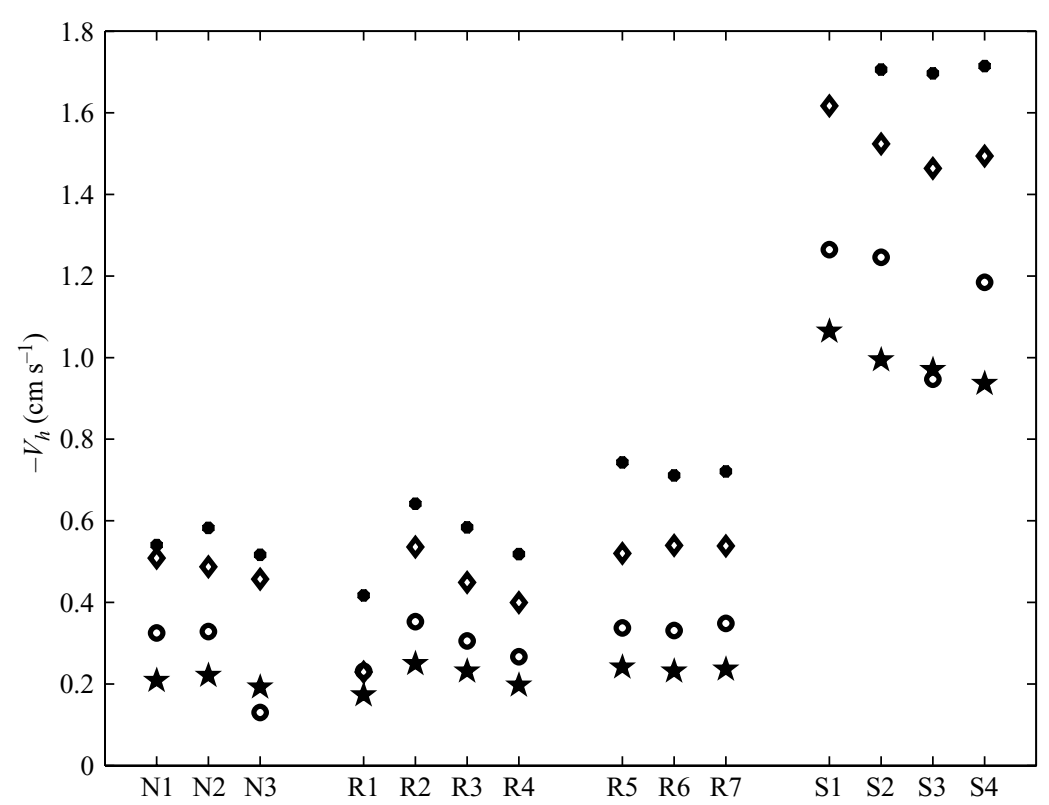

FIgURE 6. The vertical descent velocities $-V_{h}$ (along the axis of rotation) arranged by experiments. The data are marked for ball B1 by circles $(\bigcirc)$, B2 by diamonds $(\diamond)$, B5 by pentagrams $(\star)$, B6 by dots $(\bullet)$.
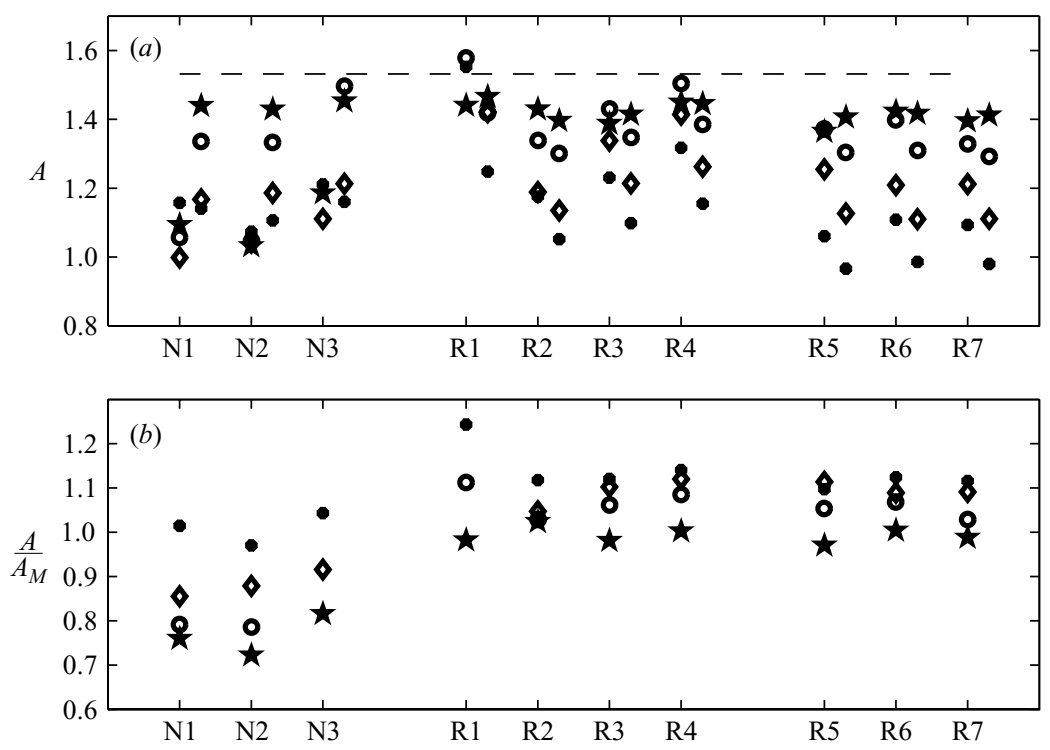

FIGURE 7. (a) The magnitude factor for the drag in the experiments with rotation: N1-N3, R1-R7. The data are marked for ball B1 by circles $(\bigcirc)$, B2 by diamonds $(\diamond)$, B5 by pentagrams $(\star)$, B6 by dots $(\bullet)$. For each experiment two data points are shown: just above the experiment marker are the values calculated from observed velocities and densities of the balls, slightly to the right are the data interpolated from the Maxworthy (1970) results. The horizontal dashed line corresponds to the discrepancy between Maxworthy's results and Stewartson's formula in the limit of $\mathscr{T} \gg 1$ and $R o \ll 1, A=1.53$. (b) The ratio $A / A_{M}$ of our results to those of Maxworthy (1970). The data are marked in the same way. 
$C_{D M}=2.60 / R o$. Expressed in terms of the drag coefficient, the Stewartson's result (5.1) gives $C_{D S}=(16 / 3 \pi) / R o$, which is also smaller by the factor of 1.53 . The reason for this discrepancy is unclear. By interpolating Maxworthy's results for $A$ as a function of $\log (\mathscr{T}), \log (R o)$ for the values of $\mathscr{T}, R o$ corresponding to our rotating experiments, these values are plotted in the same figure 7(a) by slightly shifting them to the right. One can see that there is a good agreement between our values of $A$ and those of Maxworthy's $A_{M}$ : the agreement is best for slower balls; for the fastest ball B6 the discrepancy is about $10 \%$. The horizontal dashed line indicates the limiting value of 1.53 . The slower ball B5 apparently tends to this limit while the faster ball B6 gives a value closer to unity. In other words, the linear theory of Stewartson gives the most accurate prediction for the magnitude of the drag for small Rossby numbers: for the ball $\mathrm{B} 6$ in $\mathrm{R} 1-\mathrm{R} 7 R o_{\|} \simeq 0.3-0.4$.

In figure $7(b)$ the ratio of our $A$ to that of Maxworthy, $A_{M}$, is plotted. For the centre-rotation experiments, R1-R7, the agreement is particularly good: $A / A_{M}$ is near 1.0 for slow balls and is slightly higher 1.1 for faster balls. For the tilted tank series $\mathrm{N}$ the ratio $A / A_{M}$ is about $20 \%$ smaller. Maxworthy (1968) noted that the runs where a sphere wandered off the axis of rotation produced drag coefficients somewhat below the general trend of the 'well-behaved' case. In our N-series the off-axis motion is caused by the centrifugal force which may explain the slight reduction of $A / A_{M}$. Perhaps this is related to a partial disruption and tilting of the Taylor column as observed by Hide et al. (1968), a subject deserving further study.

\section{Conclusions}

A set of experiments tracing the paths of small spheres in a tilted rotating tank has shown that the angles predicted by Stewartson's theory agree reasonably well with the observations. For the tilt angle $\alpha=30^{\circ}$, the spheres descend at an angle of $16.10^{\circ}$ in the radial direction, while azimuthally they lag behind the rotation at an angle close to the $20.18^{\circ}$ predicted by the theory. The angles of descent of the spheres are independent of the density difference between the spheres and the ambient fluid. For the motion perpendicular to the axis of rotation, the angle $-\theta_{r \lambda}$ is more sensitive to variation in the Rossby number and it shows some decrease from $45^{\circ}$ to $35^{\circ}$ as $R o_{\|}$ increases compared to the predicted $38.15^{\circ}$. The agreement is best for $R o_{\|} \simeq 0.2$, and not for infinitesimal $R o_{\|}$.

The magnitude of the velocity is linked to the density difference through the force balance acting on the spheres. A magnitude coefficient $A$ has been introduced to compare the observed velocities with those of Stewartson's theory and observations recorded by Maxworthy. It was found that $A$ lies between 1 and 1.6, indicating that the descent velocity predicted by Stewartson's theory is up to $60 \%$ larger than the observed velocities, implying that the actual drag on the spheres is larger than expected from theory. Again the prediction is most accurate for small Rossby numbers.

The reason for the discrepancy is unclear. Perhaps it is related to the failure of the linear theory to take into account that some of the fluid is trapped within the Taylor slug and travels with the sphere and also that the Taylor slug is tilted when the sphere moves perpendicular to the axis of rotation. Perhaps the 'effective body' travelling through rotating fluid and generating the inertial (gyroscopic) waves (which cause the increased drag) is different from a sphere. To answer this question direct measurements of the radiated inertial waves would be required. 
This work was supported by the National Science Foundation grant OCE-0116910. Many of the experiments were carried out during the GFD WHOI summer program 2002, and supported by a GFD fellowship.

\section{Appendix. Estimation of densities of the spheres}

In order to achieve a small descent velocity and thus a small Rossby number flow for the chosen value of the angular velocity, the density difference $\Delta \rho$ between the ball and water should be very small $O\left(10^{-3}\right)$. It is impossible to calculate the density of each ball with such accuracy directly by weighing it and measuring its volume. Instead, an indirect method is used, based on measuring the terminal descent velocity in non-rotating fluid for which case much experimental data are available. When a sphere descends in a fluid at constant speed $V$, the Archimedes force (proportional to $\Delta \rho$ ) acting on it is balanced by the hydrodynamic drag:

$$
\frac{4}{3} \pi a^{3} \Delta \rho g=\frac{1}{2} \rho V^{2} \pi a^{2} C_{D}(R e),
$$

which is usually expressed in terms of the drag coefficient $C_{D}$ dependent only on a Reynolds number, $R e=2 \mathrm{Va} / \mathrm{v}$. For the range $20 \leqslant R e \leqslant 260$ appropriate to our experiments Clift, Grace \& Weber (1978, Chap. 5, Table 5.2(C)) recommend a 'standard drag curve' in the form

$$
C_{D}=\frac{24}{R e}\left(1+0.1935 R e^{0.6305}\right) .
$$

The first term in the sum (A 2) represents the Stokes law with the drag proportional to $V$ in the linear viscous case $R e \ll 1$. The second term describes the increase of the drag due to inertial reaction of the oncoming flow with subquadratic dependence on $V$.

Observed descent velocities $V_{h}$ for all $\mathrm{N}$, R, and S experiments are shown in figure 6; they are arranged by experiment, with different balls indicated by different markers. Note that in the tilted tank experiments $\mathrm{N}$ the vertical component of velocity $V_{h}$ is determined by the same balance as in the R-series (5.7). It is clearly seen that the rotation substantially slows down the descent velocity. In the figure the rotation rate reduces from left to right. Several apparent outliers with anomalously slow descent velocity (specifically, N3-B1, R1-B2, S1-B6, S3-B1) were caused by air bubbles trapped at the surface of a nylon ball during its entry into the fluid. Visual inspection at the end of the experiment did confirm the presence of the air bubbles in these cases. When it was tried keeping the balls constantly under water during the spin-up as a potential remedy, another difficulty occurred: air bubbles started to appear at the surface of nylon due to adsorption (the effect of condensation of gases on the surfaces of solids); it was decided to abandon this route.

The variation of temperature in the laboratory in the range from $24^{\circ} \mathrm{C}$ to $26^{\circ} \mathrm{C}$ caused small but noticeable effects on the density estimation as a result of the dependence of the viscosity of the fluid on temperature and the differential thermal expansion of nylon with respect to the fluid. The viscosity correction due to temperature variation was calculated using the formula

$$
v=v_{25}\left[1+\frac{1}{v} \frac{\partial v}{\partial T}\left(T_{F}-25^{\circ} \mathrm{C}\right)\right]
$$

where the values of kinematic viscosity $\nu_{25}$ and the thermal coefficient of kinematic viscosity at $25^{\circ} \mathrm{C}$ for $15.79 \% \mathrm{NaCl}$ aqueous solution were interpolated based on 
the data taken from Lide (2002); they are listed in table 2. The values of $\nu_{25}$ in the laboratory were also checked by the Cannon Fenske Routine. The variation of $1{ }^{\circ} \mathrm{C}$ from one experiment to another during the $\mathrm{S}$ series contributed about $2 \%$ variation of $\nu$, which, in turn, affected $C_{D}(R e)$ in (A 1) and hence the estimate of $\Delta \rho$.

After calculating $\Delta \rho$ based on (A 1) for the temperature recorded during each experiment (table 1), the density anomaly was then reduced to the standard temperature of $25^{\circ} \mathrm{C}$ according to

$$
\Delta \rho_{25}=\Delta \rho+\rho_{25}\left(\alpha_{N}-\alpha_{F}\right)\left(T_{F}-25^{\circ} \mathrm{C}\right),
$$

where the value of the coefficient of thermal expansion $\alpha_{F}$ for $15.79 \% \mathrm{NaCl}$ solution was again interpolated based on the data from Lide (2002). The coefficient of cubical thermal expansion of Nylon $\alpha_{N}$ has the largest uncertainty. The manufacturer reported only the linear expansion coefficient at $20^{\circ} \mathrm{C}$, from which $\alpha_{N}=265 \times 10^{-6}{ }^{\circ} \mathrm{C}^{-1}$ was estimated as three times the linear one, though it is understood that the nylon is an anisotropic material. This value, however, was confirmed by our observation that when the temperature in our laboratory later dropped to $19.4^{\circ} \mathrm{C}$, the ball $\mathrm{B} 1$ became neutrally buoyant. During the spin-up, which normally lasted about 30 minutes, the balls were in thermal contact and had supposedly reached the same temperature as the fluid, hence $T_{F}$ in (A 4) represents both the temperature of the ball and the fluid. For a $1{ }^{\circ} \mathrm{C}$ increase in temperature $\Delta \rho$ increases about $15 \%$, making balls sink faster. Thus, the differential thermal expansion has bigger effect than viscosity.

Excluding the outliers S1-B6 and S3-B1, the averaged density anomaly of each ball was calculated at $25^{\circ} \mathrm{C}$ in experiments $\mathrm{S} 1-\mathrm{S} 4$ to be $(0.88,1.24,0.62,1.50)$ $\times 10^{-3} \mathrm{~g} \mathrm{~cm}^{-3}$ for B1, B2, B5, B6, respectively. Based on our ability to record temperature with $\pm 0.1^{\circ} \mathrm{C}$ precision and also a natural scatter of the results, the relative error of the density anomalies is estimated to be approximately $\pm 3 \%$ or absolute error $\pm 0.03 \times 10^{-3} \mathrm{~g} \mathrm{~cm}^{-3}$ The water absorption by Nylon can also contribute to $\Delta \rho$, but before the experiments the balls were stored in the salty water for several days and so that some equilibrium could be reached.

\section{REFERENCES}

Bush, J. W. M., Stone, H. A. \& Bloxham, J. 1995 Axial drop motion in rotating fluids. J. Fluid Mech. 282, 247-278.

Bush, J. W. M., Stone, H. A. \& Tanzosh, J. P. 1994 Particle motion in rotating viscous fluids: Historical survey and recent developments. Current Topics Phys. Fluids 1, 337-355.

Clift, R., Grace, J. R. \& Weber, M. E. 1978 Bubbles, Drops, and Particles. Academic.

Fletcher, R. I. 1972 The apparent field of gravity in a rotating fluid system. Am. J. Phys. 40, 959-965.

Greenspan, H. P. 1990 The Theory of Rotating Fluids. Breukelen.

Hide, R. \& Ibbetson, A. 1966 An experimental study of 'Taylor columns'. Icarus 5, 279-290.

Hide, R., Ibbetson, A. \& Lighthill, M. J. 1968 On slow transverse flow past obstacles in a rapidly rotating fluid. J. Fluid Mech. 32, 251-272.

Hocking, L. M., Moore, D. W. \& Walton, I. C. 1979 The drag on a sphere moving axially in a long rotating container. J. Fluid Mech. 90, 781-793.

Lide, D. R., (Ed). 2002 CRC Handbook of Chemistry and Physics 82nd Edn, CRC Press.

LOPER, D. E. 2001 On the structure of a Taylor column driven by a buoyant parcel in an unbounded rotating fluid. J. Fluid Mech. 427, 131-165.

Mason, P. J. 1975 Forces on bodies moving transversely through a rotating fluid. J. Fluid Mech. 71, 577-599.

Maxworthy, T. 1968 The observed motion of a sphere through a short, rotating cylinder of fluid. J. Fluid Mech. 31, 643-655. 
Maxworthy, T. 1970 The flow created by a sphere moving along the axis of a rotating, lightlyviscous fluid. J. Fluid Mech. 40, 453-479.

Moll, H.-G. 1973 The free motion of a sphere in a rotating fluid. Ing. Arch. Bd. 42, 215-224.

Moore, D. W. \& Saffman, P. G. 1968 The rise of a body through a rotating fluid in a container of finite length. J. Fluid Mech. 31, 635-642.

Moore, D. W. \& SAFFman, P. G. 1969 The stucture of free vertical shear layers in a rotating fluid and the motion produced by a slowly rising body. Phil. Trans. R. Soc. Lond. A 264, 597-634.

Pickart, R. S., Torres, D. J. \& Clarke, R. A. 2002 Hydrography of the Labrador Sea during active convection. J. Phys. Oceanogr. 32, 428-457.

Pritchard, W. G. 1969 The motion generated by a body moving along the axis of a uniformly rotating fluid. J. Fluid Mech. 39, 443-464.

Proudman, J. 1916 On the motion of solids in liquids possessing vorticity. Proc. R. Soc. Lond. A 92, 408-424.

RIEMENSCHNEIDER, U. 2002 Ball release experiment in a centrifuge. In Bounds on Turbulent Transport. 2002 Summer Study Program in Geophysical Fluid Dynamics, pp. 194-213 (available at http://gfd.whoi.edu/proceedings/2002/PDF/Riemenschneider.pdf).

SHEREMET, V. A. 2004 Laboratory experiments with tilted convective plumes on a centrifuge: a finite angle between the buoyancy force and the axis of rotation. J. Fluid Mech. 506, 217-244.

Stewartson, K. 1953 On the slow motion of an ellipsoid in a rotating fluid. Q. J. Math. Appl. Mech. 6, $141-162$.

Tanzosh, J. P. \& Stone, H. A. 1994 Motion of a rigid particle in a rotating viscous flow: an integral equation approach. J. Fluid Mech. 275, 225-256.

TAYLOR, G. I. 1917 Motion of solids in fluids when the flow is not irrotational. Proc. R. Soc. Lond. A 93, 99-113.

TAYLOR, G. I. 1921 Experiments with rotating fluids. Proc. R. Soc. Lond. A 100, 114-121.

TAYLOR, G. I. 1922 The motion of a sphere in rotating liquid. Proc. R. Soc. Lond. A 102, 180-189. 